\title{
The Natalnet Laboratory
}

\author{
Lourena Rocha, Rummenigge Dantas, \\ Julio Cesar Melo, Bruno Marques, \\ Ícaro da Cunha, Samuel Azevedo and \\ Luiz Marcos G. Gonçalves \\ Department of Computer Engineering and Automation \\ Federal University of Rio Grande do Norte \\ Natal-RN, Brazil \\ http://www.natalnet.br
}

\begin{abstract}
This paper presents the Natalnet Laboratory, a lab that conducts cutting-edge research in the areas of virtual reality, robotics and computer vision. The lab also focuses on developing innovative applications that integrate these areas. Here one can find the main research lines and ongoing projects of Natalnet Lab. Profiles of candidates as well as collaborators interested in joining the Natalnet family are also presented.
\end{abstract}

Keywords: virtual reality, augmented reality, 3D video, computer vision

\section{INTRODUCTION}

The Natalnet Laboratory is a research laboratory at the Federal University of Rio Grande do Norte. It was created in 1998 at the end of a research project funded by RNPProTem/CNPq. Today, we are part of the Department of Computer Engineering and Automation.

Coordinated by Professor Luiz Marcos G. Gonalves, the lab's main areas of interest are: Virtual Reality, Computational Vision, Robotics, 3D video, Digital TV, Multimedia and Embedded Systems.

Our mission is to contribute to the development of excellence in human resources, by promoting cutting-edge research. Thus, we encourage scientific and social advances.

We currently have 4 faculty, about 11 doctoral students, 12 master students and over 30 undergrads. Most students have scholarship.

Significant collaborations are the basis of the Natalnet Laboratory. Since its foundation, the lab has cooperated on several projects with other research groups. We have a continuos collaboration with the following groups: LAVID/UFPB [1], LCG/UFRJ [2], LaCCAN/UFAL [3], Robotics and Computacional Intelligence Laboratory/IME [4], Visgraf/IMPA [5], TeleMidia/PUC-Rio [6] and LNCC [7]. Also, we cooperate with brazilian companies such as Dynavideo [8] and Roboeduc [9]. The latter was initially incubated by our lab. Among international groups we can mention the cooperation with GVIL / University of Maryland [10].

We are interested in candidates of science and computer engineering or related fields. They must know how to program (in C language, preferably). Strong mathematical skills are recommended but not mandatory. Mostly, they should be excited about developing innovative results and applications.
Research groups interested in developing applications that integrate the fields of Virtual Reality and Computer Vision would be great collaborators. Also, we would appreciate a cooperation with labs concerned with development of softwares that integrate techniques of Virtual Reality and Robotics for education purposes.

In the next sections we will present the objectives, main research lines and ongoing projects in our lab.

\section{LAB OBJECTIVES AND RESEARCH LINES}

In a broad sense, our research objective is to create new scientific computing techniques, tools, and softwares that provide solutions to problems affecting various aspects of human life. We focus on solutions that integrate two or more of our research lines.

The Natalnet laboratory focuses on six main research lines:

- Virtual and Augmented Reality: Focusing on solutions that allow creation and maintenance of virtual environments and its many aspects, this research line is one of the most mature that we have - counting with many projects approaching from simple virtual environments to augmented reality.

- Computer Vision: In parallel with the virtual and augmented reality, the computer vision line groups a series of mathematical representation aiming to solve the main problem of extracting information using 2D images. The main goal here is to establish a way to map real environments to virtual with the cheapest solution available.

- Robot Systems: Supporting the computer vision line, we work with robotic systems. Robots with vision systems are an automatized way to extract spacial information in any environment. Using computer vision algorithms, networked systems and microelectronics we developed simple robots, teleoperation and multi-modal systems.

- 3D Videos: We are interested in improve the pipeline of 3D videos creation. Mainly, we focus on the problems of $3 \mathrm{D}$ reconstruction and the $3 \mathrm{D}$ video representation. Our goal is to use low-cost technology and develop new techniques to propose cutting-edge solutions to such problems. 


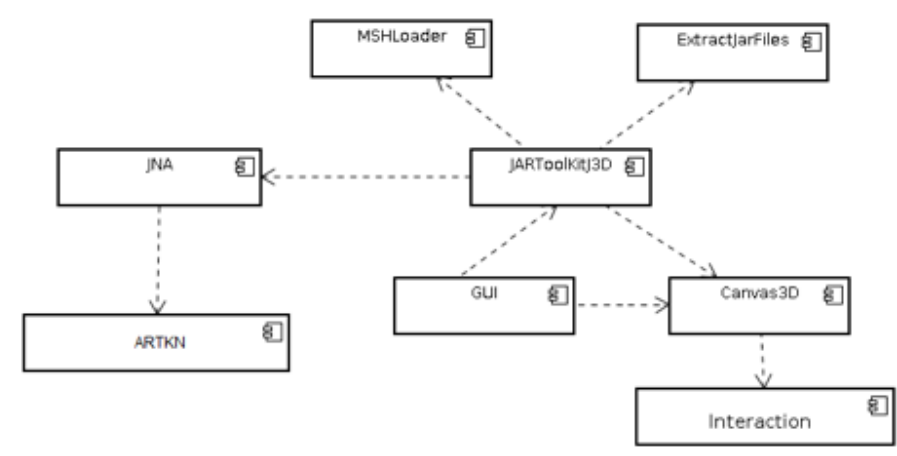

Fig. 1. GTRM Architecture.

- Digital Television: This research line focus on developing complete solutions and subsystems aiming the recent created Brazilian digital TV system. In recent researches and projects we had worked aiming to easy the creation of content to the Brazilian standard and to bring up new techniques of human-computer interaction to improve the viewer interaction.

- Embedded Systems: Aiming for solutions that cover the last four research lines, this one is still in its very beginning in our laboratory. However we have had results merging computer vision and hardware constructs in order to accelerate the processing of bigger images. The main goal of this research line is to improve the results of the main lines by developing hardwired solutions.

\section{Lab Ongoing Projects}

This section describes some of the projects being developed by the Natalnet group.

\section{A. GTRM}

The use of augmented reality allows to enhance the performance of a presentation, using 3D models that are displayed superimposed on the presentation environment. We can see the augmented reality as a new tool in support of the teaching activity as it enhances the perception of students and emphasizes information that is not perceived directly by the use of their own senses.

In this context, we execute the GTRM project that aims the creation of a system that should be easy to use for teachers with little knowledge about computers and want to use augmented reality techniques to improve their classes [11].

Figure 1 shows the project modules, the system is developed over the ARToolkit API [12] that provides a full AR solution together with OpenGL and a suitable video capture engine. We created two user interfaces one to be be used by the teacher and other to the students both adaptations of the ARToolkit. We tested the system implementing a use case, a class of chemistry, Figure 2 shows the use case implemented on the student's user interface.

\section{B. GTMV}

Technical professionals such as experts in graphical design are generally requested in order to create a virtual

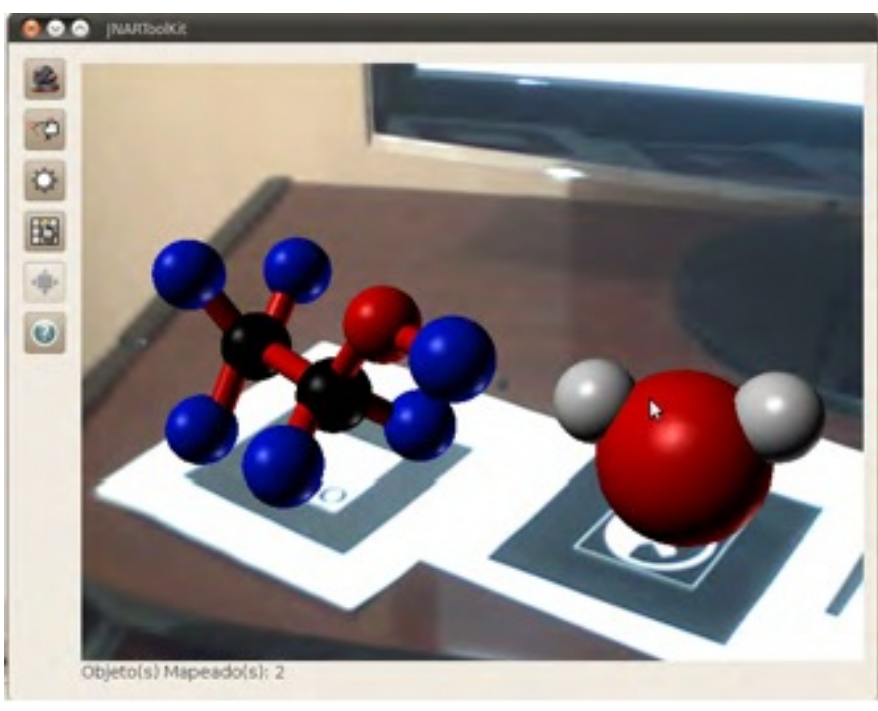

Fig. 2. GTRM: User interface with chemistry class.

museum. These professionals act on the design and also on the maintenance of these museums. Generally, the curator of the museum only decides where the artworks will be set on the virtual version of the museum and all of the hard work is passed to those professionals. On one hand, this is nice because the curator does not have to worry about learning new technologies in order to create a museum. On the other hand, there is a need of these professionals. In fact, if one could allow the curator to make himself the tech job, in an easy fashion, so any person without any knowledge of computer graphics could build and edit a museum. The graphics design work would not be boring.

To minimize the need of tech professional during the creation, edition and visualization of virtual museum, we have built in the project GTMV a system that joined the virtual multi-user environments paradigm and some easy-touse authoring tools, which we have also developed.

The project was build focusing on web-services in order to enable easy access to the tools. The system architecture in Figure 13 shows that it consists of a main Web System that is the access point for the users that can be either curators or visitors. The Curator uses the curator interface to build his museum from previous loaded 3D models or upload his own. He could also test different layouts of artworks that are loaded in the database by using the museum editor tool. The visitor will use the web site and use the visitor interface to access a 3D multiuser version of the registered museums. See [13] for details.

In fact there are other systems that could be compared with ours. However most of them do not allow the multi-user interface neither 3D view of the museums. In this project we had developed many other tools like the guide editor [14] that allows curators to create automatic guides for their museum; properly 3D format to be flexible with the 3D technologies used; 3D environment editors and others. Figure 4 shows the first museum modeled by our system. 


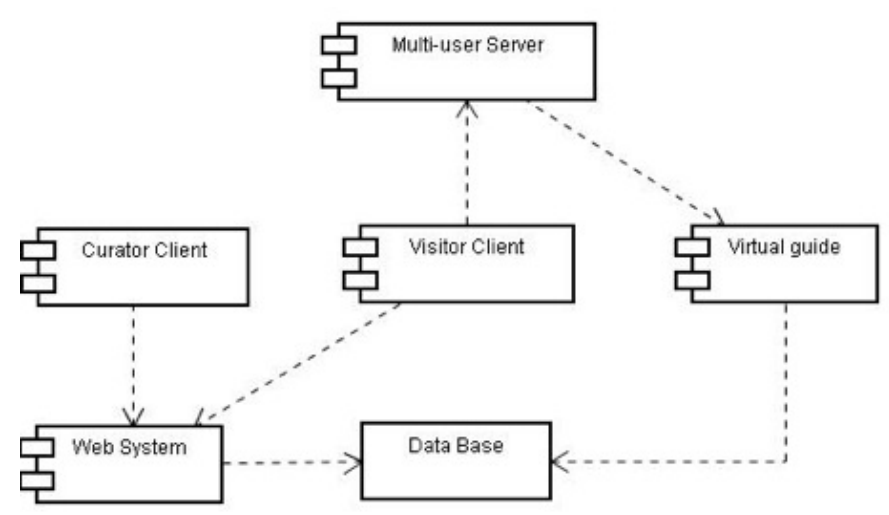

Fig. 3. GTMV Architecture.

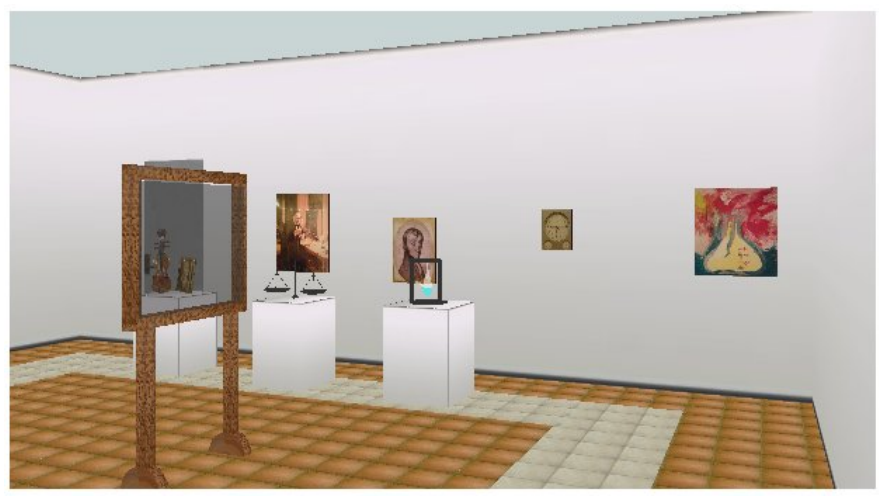

Fig. 4. First museum modeled by our GTMV system: Museum of the Nucleus of Art and Culture (NAC) at UFRN.

\section{3D Videos}

Nowadays, the Natalnet laboratory has several ongoing projects in three-dimensional area. To allow such development, the laboratory has a 3D Scanner HD by NextEngine, two Minoru cameras by Novo, two Kinects by Microsoft, a Point Gray Bumblebee XB3 stereo camera, a Panasonic 3DAG1P professional 3D camera and a JVC GD-463D10 3D monitor. See Figure 5.

1) $3 D$ reconstruction based on sensor fusion: There are many methods to obtain scene depth information. They are usually categorized in two mainly classes: passive and active. Depending on the range, sensors are called passive or active, respectively.

One of the most popular and well established is probably stereo vision [15]. One of the reasons for that is the low cost of a stereo system. Despite the advances in stereo methods, problems such as occlusion and textureless are still hard to deal with.

On the other hand, Time-of-Flight camera (ToF camera) is an active range sensor that creates distance data with help of the time-of-flight (TOF) principle. The time-of-flight camera is a class of flash-imaging LIDAR, in which the entire scene is captured with each laser or light pulse, rather than being scanned point-by-point with a moving laser as in scanning. They can capture dynamic scenes at real-time frame rates and

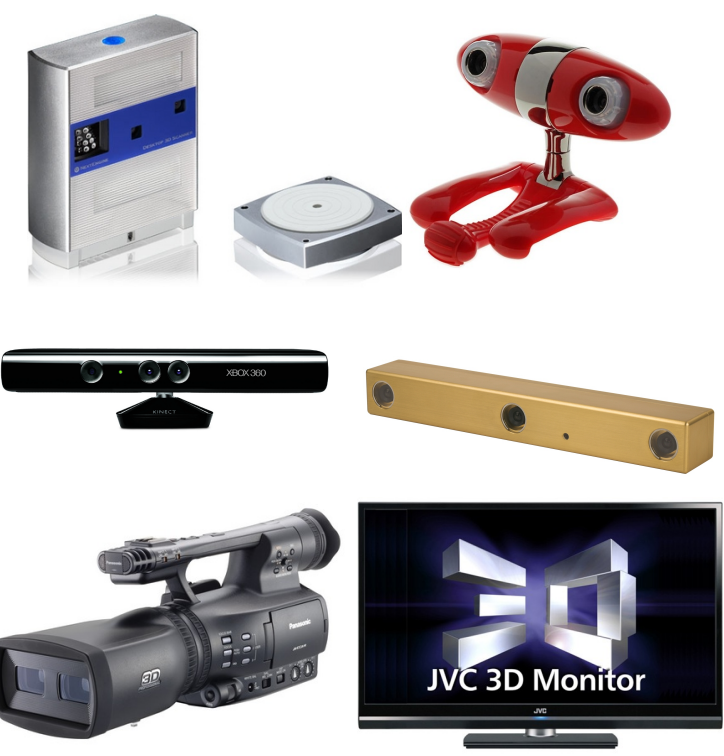

Fig. 5. Lab equipments used for $3 \mathrm{D}$ research

can exceed passive stereo on textureless regions and repeated patterns. However, the depth maps returned from the ToF sensor are commonly in low resolution.

The work in development at Natalnet laboratory, intend to improve depth maps by combining stereovision methods with depth captured by ToF cameras. The system is low cost and aims to improve depth maps of dynamic scenes.

2) Representation of $3 D$ videos: During the first stage of this work, we have studied approaches to the 3D video representation problem. Our current stage is to work on the Social Snapshot system, a system that tries to enable spacetime 3D photography using mobile devices, assisted by a his auxiliary sensors and networking features. The end result of pipeline is a set of locally optimized 2.5D meshes (one for each photo entry). While these models may be globally inconsistent, the system presents a navigation model that uses information retrieved from the camera and pose interpolation techniques to navigate the reconstruction interactively. We propose in this stage to extend this system so the transition between poses can be done more smoothly, and increase their ability to capture. The study of techniques to be incorporated into the system will be of great value because these techniques can also be incorporated in the final stage of the $3 \mathrm{D}$ video representation work.

3) Structure from Motion Based Visual Odometry: Aiming mobile robots, we envisioned and designed a Visual Odometry system, offering a low cost and fast localization method to allow accurate position estimation in unstructured environments for robotic systems. Instead of employing expensive sensors such as Lasers, Lidars or Time of Flight Cameras, a single RGB camera is used as the only sensing device of the system.

State of the art Computer Vision algorithms and currently available computer processing power are thus exploited to enable a full 6DOF localization system based solely on images 


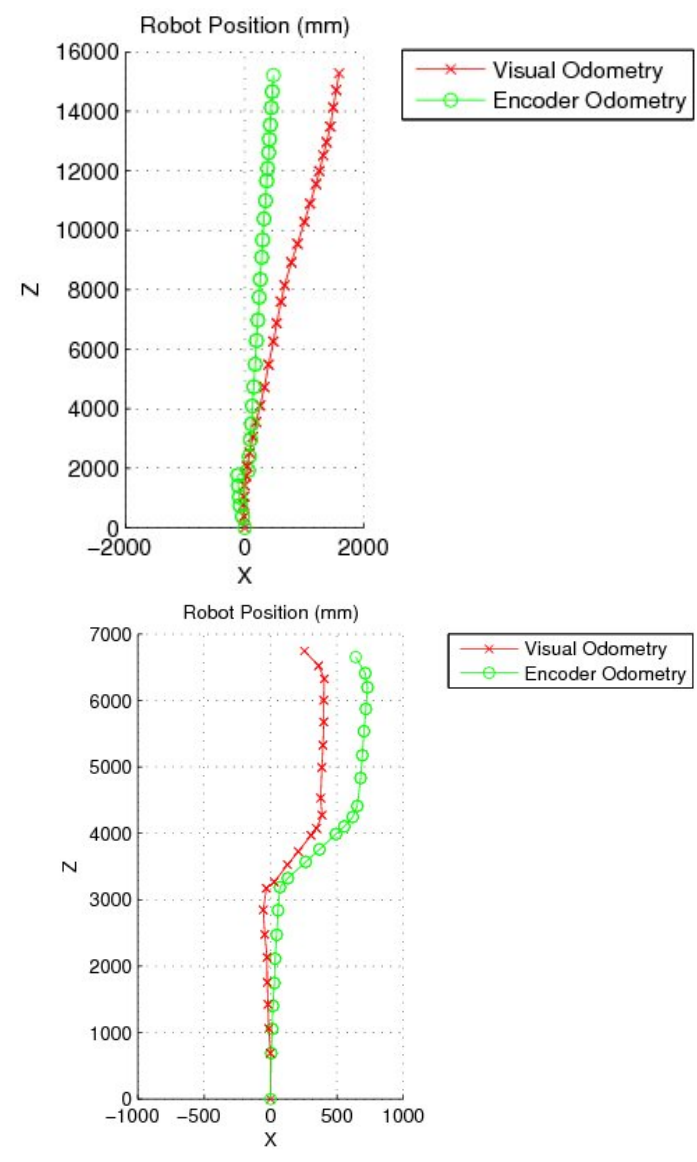

Fig. 6. Results for two different images.

of the operation environment.

Particularly, we have chosen to build our software grounded on the theory of Structure from Motion systems, mainly because this class of solutions are less restrictive than Simultaneous Localization and Mapping (SLAM) approaches.

Hence, relative position estimates are evaluated at each acquired image, through a proccess that involves matching image features across consecutive frames, finding the relative camera poses between image pairs and obtaining a sparse representation of the visualized scene (3D reconstruction). See Figure 6.

Ultimatey, at each time step the system offers a reliable representation of the world and a estimate of the mobile platform position and orientation.

\section{CONCLUSION}

In this paper we introduce the laboratory Natalnet, with our main areas of activity, research interests and major ongoing projects in the fields of Virtual Reality, Augmented Reality and $3 \mathrm{D}$ video.

Although the GT-MV project achieved its main objectives there are still many improvements to perform. We had evaluated the system usage using questionnaires. The tests showed that our system is fully functional but it still needs improvements on usage. We have to simplify the museum management and even the navigation across the 3D environments. Also, we still don't have an easy way to convert real museums to 3D presentations what will be required in most cases. The GT-MV project will continue to evolve in these and other aspects.

The GT-RM project is still very young. Its main objectives were achieved. However, we do not have a complete evaluation of its usage. Another problem that we are facing when developing augmented reality (AR) systems is how to generate AR content. For the GT-RM system is easy to add the 3D contents on the system but how the teacher will generate and link those contents with the whole class content? These and other questions are leading the next efforts in the GT-RM project in future developments.

Future directions of research in the project of Structure from Motion based on Visual Odometry will comprise loop closure detection, allowing mobile robots to self re-localizing within the environment.

\section{ACKNOWLEDGMENT}

The authors would like to thank the Rede Nacional de Ensino e Pesquisa (RNP), Conselho Nacional de Desenvolvimento Científico e Tecnológico (CNPQ), Coordenação de Aperfeiçoamento de Pessoal de Nível Superior (CAPES), Financiadora de Estudos e Projetos (FINEP) and Ministério da Cultura (Minc) for supporting the projects mentioned in this paper.

\section{REFERENCES}

[1] (2011) The Lavid website. [Online]. Available: http://www.lavid.ufpb.br/

[2] (2011) The LCG website. [Online]. Available: http://www.lcg.ufrj.br/

[3] (2011) The LaCCAN website. [Online]. Available: http://www.ufal.edu.br/unidadeacademica/ic/pesquisa/grupos/laccan

[4] (2011) The Laboratório de Robótica e Inteligência Computacional website. [Online]. Available: http://www.comp.ime.eb.br/ robotica/

[5] (2011) The Visgraf website. [Online]. Available: http://www.visgraf.impa.br/

[6] (2011) The TeleMídia website. [Online]. Available: http://www.telemidia.puc-rio.br/

[7] (2011) The LNCC website. [Online]. Available: http://www.lncc.br/

[8] (2011) The Dynavideo website. [Online]. Available: http://www.dynavideo.com.br/

[9] (2011) The RoboEduc website. [Online]. Available: http://www.roboeduc.com/

[10] (2011) The GVIL website. [Online]. Available: http://www.cs.umd.edu/gvil/

[11] L. Farias, R. Dantas, and A. Bulamaqui, "Educ-ar: A tool for assist the creation of augmented reality content for education," in Proc. IEEE VECIMS'2011, 2011, pp. 1-5.

[12] T. H. Lab. (2011) The ARToolKit home page. [Online]. Available: http://www.hitl.washington.edu/artoolkit/

[13] R. R. Dantas, A. M. F. Burlamaqui, S. O. Azevedo, J. C. P. Melo, A. A. Souza, L. Gonçalves, C. A. Schneider, J. Xavier, and L. Farias, "Gtmv: Virtual museum authoring systems," in Proc. IEEE VECIMS'2009, 2009, pp. 129-133.

[14] R. R. Dantas, J. C. P. de Melo, J. Lessa, C. A. Schneider, H. Teodósio, and L. M. G. Gonçalves, "A path editor for virtual museum guides," in Proc. IEEE VECIMS'2010, 2010, pp. 136-140.

[15] E. Trucco and A. Verri, Introductory Techniques for 3-D Computer Vision. Prentice Hall, 1998. 